\title{
Juxtamembrane-type c-kit gene mutation found in aggressive systemic mastocytosis induces imatinib-resistant constitutive KIT activation
}

\author{
Nami Nakagomi ${ }^{1,2}$ and Seiichi Hirota ${ }^{1}$
}

Aggressive systemic mastocytosis (ASM) is a very rare form of mast cell neoplasm that does not benefit from conventional chemotherapy. The majority of adult mast cell neoplasms and gastrointestinal stromal tumors (GISTs) have mutations in the proto-oncogene c-kit, which encodes the KIT receptor tyrosine kinase. The c-kit gene mutations are generally confined to the tyrosine kinase II domain in mast cell neoplasms, but are often observed at the juxtamembrane domain in GISTs. We found a case of ASM with a juxtamembrane-type mutation, Val559lle, and in this report the mutation was characterized through transfection of the mutated c-kit cDNA into human embryonic kidney cells. Phosphorylation of KIT and its possible downstream signaling molecules were examined in the presence or absence of imatinib, a selective tyrosine kinase inhibitor. Ligand-independent autophosphorylation was observed in the mutant KIT with Val559lle as well as that with Val559Asp, as found in GISTs. Imatinib, at a concentration of $10 \mu \mathrm{M}$, inhibited autophosphorylation of the mutant KIT with Val559Asp, but not that with the Val559lle. Phosphorylation of MAPK and STAT5 was also inhibited by imatinib at the same concentration, in cells expressing Val559Asp but not in those expressing Val559lle. These results suggest that different mutations, even at the same codon, in juxtamembrane domain of the c-kit gene show different inhibitory effects of imatinib, and that patients with GISTs or mast cell neoplasms possessing this Val559lle mutation are resistant to imatinib therapy.

Laboratory Investigation (2007) 87, 365-371. doi:10.1038/labinvest.3700524; published online 29 January 2007

KEYWORDS: c-kit gene; gain-of-function mutation; juxtamembrane-type; systemic aggressive mastocytosis; imatinib

As defined in WHO's classification, aggressive systemic mastocytosis (ASM) is a subvariant of systemic mastocytosis which is characterized by abnormal growth and accumulation of mast cells in one or more organs with end-organ impairment. ${ }^{1}$ In most patients with ASM, conventional therapy is not effective and their prognosis is extremely poor. ${ }^{2}$ Recent advances in understanding the biology of this tumor using gene analysis revealed that the majority of adult mast cell neoplasms including ASM had mutations in proto-oncogene c-kit. ${ }^{3}$

The c-kit gene encodes a type III receptor tyrosine kinase (TK) KIT, ${ }^{4}$ the ligand of which is stem cell factor (SCF). ${ }^{5}$ KIT consists of an extracellular domain with five immunoglobulin-like repeats, a transmembrane domain, a juxtamembrane domain and a TK domain split into two portions, the TK I domain and the TK II domain. The binding of SCF to KIT promotes dimerization and phosphorylation of the receptor. Then diverse intracellular re- sponses are induced through various signal transduction pathways such as RAS/MAPK, PI3K/Akt and the Jak/STAT systems. The SCF-KIT interaction is considered to be indispensable for normal regulation of differentiation and proliferation of hematopoietic progenitors, especially erythroid lineages, melanocytes, germ cells, mast cells and interstitial cells of Cajal. ${ }^{5,6}$ Thus, the loss-of-function mutations of the c-kit gene result in a deficiency of those five cell types. On the other hand, gain-of-function mutations of the c-kit gene promote constitutive phosphorylation of KIT and consequent activation of downstream molecules without the requirement of SCF-KIT interaction. These mutations are considered to be a cause of mast cell neoplasms and gastrointestinal stromal tumors (GISTs). ${ }^{3,7-10}$ In mast cell neoplasms, most of the mutations are specifically confined to the codon 816 at exon 17 encoding TK II domain. ${ }^{7-9}$ Although the two gain-offunction mutations of the c-kit gene, Val816Asp and

\footnotetext{
${ }^{1}$ Department of Surgical Pathology, Hyogo College of Medicine, Nishinomiya, Japan and ${ }^{2}$ Department of Pathology, Wakayama Medical University, Wakayama, Japan Correspondence: Dr S Hirota, MD, Department of Surgical Pathology, Hyogo College of Medicine, 1-1 Mukogawa-cho, Nishinomiya, Hyogo 663-8501, Japan. E-mail: hiros@hyo-med.ac.jp

Received 23 October 2006; revised 08 December 2006; accepted 11 December 2006
} 
Val560Gly, were first found in the mast cell leukemia cell line (HMC-1 cells), the Val560Gly mutations have been only rarely observed in the cases with indolent systemic mastocytosis. ${ }^{11}$ A rare case of systemic mastocytosis with germline transmembrane domain mutation (Phe522Cys) has also been reported. ${ }^{12}$ On the other hand, most of the mutations are observed at exon 11 encoding juxtamembrane domain in GISTs. ${ }^{10}$ The minority of GISTs have the mutations of the c-kit gene at exon 9 encoding extracellular domain, at exon 13 encoding TK I domain, or at exon $17 .^{13-15}$

Imatinib has developed as an agent to inhibit constitutive activation of BCR-ABL, which is a cause of chronic myelogenous leukemia. In vitro studies revealed that it effectively inhibited not only wild-type KIT but also juxtamembranetype mutant KIT, but not TK II-type mutant KIT, especially Asp816Val or Asp816Tyr observed in mast cell neoplasms. ${ }^{16,17}$ And the drug is now being successfully used for patients with metastatic or unresectable GISTs ${ }^{18}$ but not commonly for patients with mast cell neoplasms.

We encountered an autopsy case of ASM with a novel c-kit gene mutation at the juxtamembrane domain. The mutation was present at codon 559 and resulted in amino-acid substitution Val to Ile (Val559lle). The point mutation at codon 559 has been reported in GISTs, but the substituted aminoacid type is usually Asp (Val559Asp), Ala (Val559Ala) or Gly (Val559Gly). ${ }^{19-22}$ To our knowledge, only one case with the Val559Ile has been reported ${ }^{23}$ but biological characterization of the mutation has not been carried out. We consider that this is the first ASM case with juxtamembrane-type c-kit gene mutation. In this report, we characterized the Val559Ile KIT mutation by its transfection into human embryonic kidney (HEK) 293 cells and further compared the downstream signaling pathways of the Val559Ile with that of the Val559Asp commonly observed in GISTs.

\section{MATERIALS AND METHODS}

\section{Patient and Clinical History}

A 77-year-old Japanese man consulted a doctor with complaint of macroscopic hematuria, but examination of the urinary system revealed no causative lesions. Peripheral blood counts showed pancytopenia (white blood cells 3400/ $\mu \mathrm{L}$, hemoglobin $9.7 \mathrm{~g} / \mathrm{dl}$ and platelet $17000 / \mu \mathrm{l}$ ) and he was admitted to the hospital. Computed tomography revealed ascites, an enlarged liver with irregular surface and splenomegaly with infarction. Infiltration of hematological malignant cells into liver and spleen was suspected, but a biopsy could not be carried out owing to a tendency to bleed. In spite of prednisolon administration and platelet transfusion, his condition got worse and the patient died without a final diagnosis only 3 months after the onset of hematuria. An autopsy was performed to elucidate the cause of his death.

\section{Tissue Preparation and Immunohistochemistry}

Tissues from various organs were obtained during autopsy, fixed with formalin and embedded in paraffin. Paraffin sec- tions (3- $\mu \mathrm{m}$ thick) were used for conventional H\&E staining and immunohistochemistry. Immunohistochemistry was performed using ENVISION + KIT HRP (DAB) system (DAKO cytomation, Kyoto, Japan). Antibodies (Abs) used were listed in Table 1. Antigen unmasking was performed using appropriate retrieval techniques according to the manufacturer's instructions.

\section{Analysis of the c-kit Gene Mutation}

Genomic DNA was extracted from formalin-fixed paraffin-embedded tissues using DEXPAT (TAKARA, Kyoto, Japan). Exons 9, 11, 13 and 17 of the c-kit gene were amplified by polymerase chain reaction (PCR) with Ampli Taq Gold (PerkinElmer, Norwalk, CT, USA) using the following oligonucleotide primer pairs; for exon 9, 5'-TTCCCTTTAGATGCTCTGCTTCT-3'/ $5^{\prime}$-CCTTTGTTGTTACCTTTAAATGC-3', for exon 11, $5^{\prime}$ GAGTGCTCTAATGACTGACTGAGA-3' $/ 5^{\prime}$-AAAGGTGACATG GAAAGCCC- $3^{\prime}$, for exon 13, 5'-GCTTGACATCAGTTT GCCAG- $3^{\prime} / 5^{\prime}$-AAAGGCAGCTTGGACACGGCTTTA- $3^{\prime}$ and for exon 17, 5'-GTTTTCTTTTCTCCTCCAAC- ${ }^{\prime} / 5^{\prime}$-TGCAGGACT GTCAAGCAAGCAGAG- $3^{\prime}$. After purification of the PCR products with the QIAquick Gel Extraction Kit (QIAGEN, Valencia, CA, USA), direct sequencing was performed with ABI BigDye terminator ver.3.1 (Applied Biosystems, Foster City, CA, USA) and ABI Prism 3100-Avant Genetic Analyzer (Applied Biosystems). The study was performed under the authors' institutional guidelines and was approved by the institutional review boards.

\section{Construction and Transfection of Mutated c-kit cDNA with Val559lle}

The whole coding region of human wild-type c-kit cDNA was subcloned into expression vector pcDNA3.1 Zeo $(+)$ (Invitrogen, San Diego, CA, USA). To generate the human c-kit cDNA containing the Val559Ile mutation, site-directed

Table 1 List of antibodies used in the present study and the staining results

\begin{tabular}{lllll}
\hline Antigen & Clone & $\begin{array}{c}\text { Antigen } \\
\text { retrieval }\end{array}$ & Source & Results \\
\hline CD2 & MT910 & + & DAKO & + \\
CD25 & ACT-1 & + & DAKO & + \\
KIT (CD117) & Polyclonal (A4502) & - & DAKO & + \\
Mast cell tryptase & AA1 & + & DAKO & + \\
MITF & D5 & + & DAKO & + \\
CD68 & PG-M1 & + & DAKO & + \\
CD45 & 2B11+PD7/26 & - & DAKO & + \\
CD34 & QBEnd10 & + & Immunotech & - \\
CD3 & F7.2.38 & + & DAKO & - \\
CD45RO & UCHL-1 & - & DAKO & - \\
CD20cy & L26 & - & DAKO & - \\
CD79a & JCB117 & + & DAKO & - \\
& & & & +
\end{tabular}


mutagenesis was performed. Then SnaBI-MroI fragment (nucleotide 1140-2683) of the human wild-type c-kit cDNA in the expression vector was replaced by the corresponding fragment of the mutated c-kit cDNA with Val559Ile. The expression vector with the mutation was transfected into HEK293 cells using Mirus Trans IT-293 transfection reagent (Madison, WI, USA). Wild-type c-kit cDNA in expression vector pcDNA3.1 Zeo $(+)$ and mutated c-kit cDNA with the Val559Asp in expression vector pEF-BOS were transfected in the same way.

\section{Immunoprecipitation and Immunoblotting of KIT}

The procedures of cell lysis, sodium dodecyl sulfate-polyacrilamide gel electrophoresis and immunoblotting were performed as described. ${ }^{24}$ After the depletion of serum for $6 \mathrm{~h}$, the HEK293 cells expressing wild-type KIT, mutant KIT with Val559Asp or mutant KIT with Val559Ile were collected and incubated with or without $100 \mathrm{ng} / \mathrm{ml}$ of human recombinant SCF (PeproTec EC, London, UK) at $37^{\circ} \mathrm{C}$ for $10 \mathrm{~min}$. Cells were collected and lysed using CelLytic-M lysis buffer (Sigma-Aldrich, St Louis, MO, USA) with the addition of complete protease inhibitor cocktail tablets (Roche, Mannheim, Germany), phosphatase inhibitor cocktail (Sigma-Aldrich) and $1 \mu \mathrm{M} \mathrm{Na}_{3} \mathrm{VO}_{4}$. The extracts were precipitated with murine anti-human KIT monoclonal $\mathrm{Ab}$ (YB5.B8, Pharmingen, San Diego, CA, USA) and protein G Sepharose beads (Amersham Bioscience Corp, Piscataway, NJ, USA). Immunoblotting was performed with mouse anti-phosphotyrosine monoclonal Ab (4G10, Upstate Biotechnology, Lake Placid, NY, USA) and rabbit anti-human KIT polyclonal Ab (A4502; DAKO Cytomation). The binding of the $\mathrm{Ab}$ was detected by enhanced chemiluminescence Western Blotting Detection System (Amersham Bioscience Corp).

\section{Effect of Imatinib on Phosphorylation of KIT and Its Downstream Signaling Molecules}

After serum deprivation for $6 \mathrm{~h}$, the HEK293 cells expressing mutant KIT with the Val559Asp or Val559Ile were cultured at various concentrations of imatinib $(0,0.01,0.1,1$ and $10 \mu \mathrm{M})$ (Novartis Pharma, Basel, Switzerland) for $90 \mathrm{~min}$. Then cells were collected and lysed as described. ${ }^{24}$ Phosphorylation of KIT was detected by anti-phosphotyrosine monoclonal $\mathrm{Ab}$ (4G10) after immunoprecipitation with KIT monoclonal Ab (YB5.B8). To examine the inhibitory effect of imatinib on phosphorylation of KIT downstream signaling molecules, the cells expressing mutant KIT with Val559Asp or Val559Ile were cultured at concentrations of 0 and $10 \mu \mathrm{M}$ of imatinib for 90 min after $6 \mathrm{~h}$ serum deprivation. Phospho-specific MAPK Ab (9109, Cell Signaling Technology, Beverly, MA, USA), phospho-Akt Ab (9271, Cell Signaling Technology), phospho-STAT1 Ab (9171, Cell Signaling Technology) and phospho-STAT5 Ab (9351, Cell Signaling Technology) were used to detect phosphorylation of MAPK, Akt, STAT1 and STAT5. Immunoblotting of phospho-STAT1 and phospho-
STAT5 was carried out after immunoprecipitation using total STAT1 Ab (9172, Cell Signaling Technology) and total STAT5 Ab (9352, Cell Signaling Technology). Each membrane was subsequently reprobed with total MAPK Ab (9102, Cell Signaling Technology), pan-Akt Ab (9272, Cell Signaling Technology), total STAT1 Ab (9172) or total STAT5 Ab (9352).

\section{RESULTS \\ Macroscopic, Histological and Immunohistochemical Analysis}

At autopsy, hepatosplenomegaly (liver $3180 \mathrm{~g}$, spleen $480 \mathrm{~g}$ ) was noted. There were no space-occupying lesions in the liver or spleen, but sporadic infarcted lesions were observed in the spleen. Microscopically, short-spindled tumor cells diffusely infiltrated the spleen (Figure 1a). The tumor cells were also found in the hepatic portal area and renal interstitium. In the bone marrow, paratrabecular infiltration of the tumor cells was shown. The tumor cells exhibited metachromasia by toluidine blue staining (Figure 1b) and were immunohistochemically positive for CD25 (Figure 1c), KIT (Figure 1d), mast cell tryptase (Figure 1e) and MITF (Figure 1f). Immunohistochemical details including other markers are summarized in Table 1 . These results confirmed the diagnosis of ASM.

\section{Identification of c-kit Gene Mutation}

To examine whether the c-kit gene mutation is observed in tumor cells of ASM, genomic DNA was extracted from paraffin-embedded splenic tissues, because tumor cells diffusely infiltrated the spleen and contamination of non-neoplastic cells was considered to be minimum. As most c-kit gene mutations are observed at exon 9, exon 11, exon 13 or exon 17 in human neoplasms including mast cell neoplasms and GISTs, DNA fragments of those exons were amplified by PCR. Direct sequencing of the products revealed point mutation at codon 559 (GTT to ATT) in the exon 11 encoding juxtamembrane domain (Figure 2a), resulting in amino-acid substitution from Val to Ile. No mutations were detected at exons 9, 13 and 17. Analysis of the genomic DNA extracted from paraffin-embedded prostatic tissue without tumor cell infiltration showed no mutations in exon 11 (Figure 2b) in addition to exons 9,13 and 17.

\section{Constitutive Activation of Mutant KIT with Val559lle}

To investigate the effect of Val to Ile substitution at codon 559, the expression vector containing the mutated c-kit cDNA was transfected into HEK293 cells. The expression vector containing the wild-type c-kit cDNA or the mutated c-kit cDNA with Val559Asp observed in GISTs was also transfected into the cells, and the phosphorylation status of KIT was compared. As shown in Figure 2c, autophosphorylation of the wild-type KIT without SCF stimulation was very weak but became significant in response to SCF addition. The mutant KIT with Val559Asp was strongly 

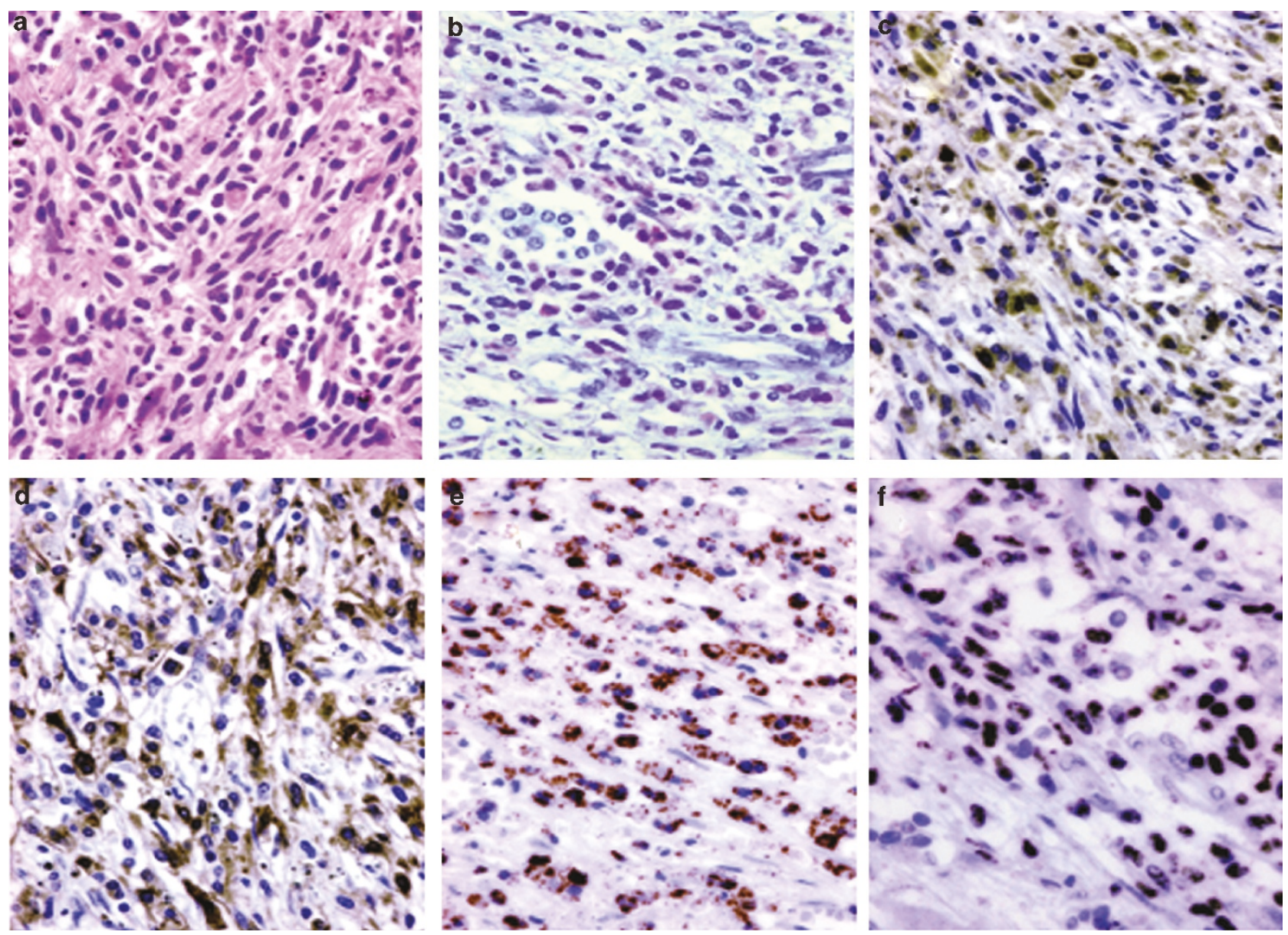

Figure 1 Histology and immunohistochemical profile of tumor cells. (a) Short spindled atypical tumor cells diffusely infiltrated in splenic tissue, hematoxylin and eosin staining. (b) Some tumor cells showed metachromasia in toluidine blue staining. (c-f) Immunohistochemistry for CD25 (c), KIT (d), mast cell tryptase (e) and MITF (f) revealed that the tumor cells are positive for all of these markers, suggesting that the tumor cells have differentiation features for mast cells. Staining of CD25 and KIT mainly shows a membranous pattern. On the other hand, staining pattern of mast cell tryptase is cytoplasmic, and that of MITF is nuclear.

autophosphorylated even in the absence of SCF (Figure 2c) as reported previously. ${ }^{10} \mathrm{~A}$ similar pattern was observed in the mutant KIT with Val559Ile (Figure 2c).

\section{Effect of Imatinib on Autophosphorylation of Mutant KIT with Val559lle}

To examine the effect of imatinib on mutant KIT with Va1559Ile, the HEK293 cells transfected with the mutated c-kit cDNA were cultured at various concentrations of the reagent $(0,0.01,0.1,1$ and $10 \mu \mathrm{M})$. As a comparison, the HEK293 cells transfected with the mutated c-kit cDNA with Val559Asp were also examined. Autophosphorylation of mutant KIT with Val559Asp was markedly inhibited at a concentration of $1 \mu \mathrm{M}$ of imatinib, but that of mutant KIT with Val559Ile was not inhibited, even at a concentration of $10 \mu \mathrm{M}$ (Figure 3).

\section{Effect of Imatinib on Phosphorylation of KIT Downstream Signaling Molecules}

To clarify the relationship between activation of KIT and that of its downstream signaling molecules, the effect of imatinib on phosphorylation of MAPK, Akt, STAT1 and STAT5 was examined in original HEK293 cells and those transfected with mutated c-kit cDNA with Val559Ile or Val559Asp. When phosphorylation of mutant KIT with Val559Asp was inhibited by imatinib at a concentration of $10 \mu \mathrm{M}$ (Figure 4a), that of MAPK and STAT5 was also inhibited significantly (Figure $4 \mathrm{~b}, \mathrm{c}$ ). In contrast, not only KIT but also MAPK and STAT5 remained phosphorylated in mutant KIT with Va1559Ile even after the addition of imatinib at a concentration of $10 \mu \mathrm{M}$ (Figure $4 \mathrm{a}-\mathrm{c}$ ). No apparent phosphorylation of STAT1 was observed in any type of HEK293 cells even without imatinib treatment (Figure 4d). Akt was strongly phosphorylated even in the original HEK293 cells, and the inhibitory effect of imatinib was not apparent in any of the cell types examined (Figure 4e).

\section{DISCUSSION}

We found a case of ASM with a juxtamembrane-type c-kit gene mutation of Val559Ile. Although HMC-1 cells isolated from a patient with mast cell leukemia have a juxtamem- 


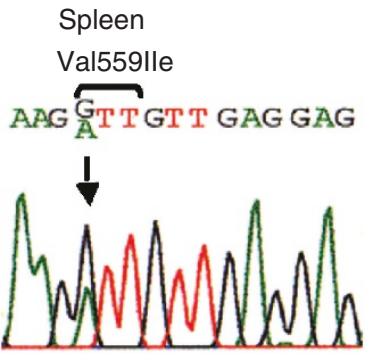

C

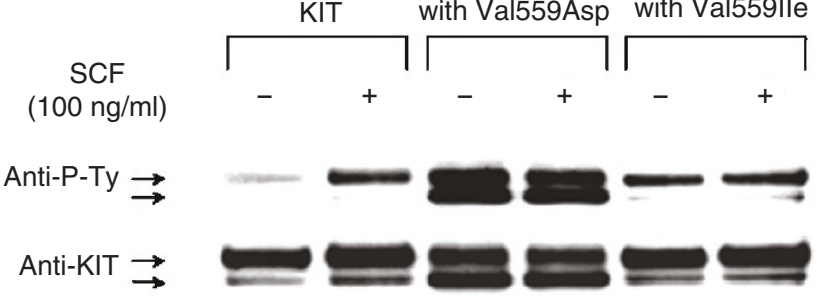

Figure 2 Detection of c-kit gene mutation and phosphorylation status of mutant KIT. (a) Direct sequencing of PCR-amplified products of genomic DNA derived from tumor cells of spleen revealed a point mutation at codon 559 (GTT to ATT). (b) Genomic DNA derived from prostatic tissue without tumor cells did not show the point mutation. (c) Immunoblotting with antiphosphotyrosine $\mathrm{Ab}$ (4G10) revealed ligand-independent autophosphorylation of mutant KIT with the Val559Asp and mutant KIT with Val559lle. Wild-type c-kit cDNA, mutated c-kit cDNA with Val559Asp or that with Val559lle was transfected into HEK293 cells, and the cells were incubated with or without SCF after serum starvation for $6 \mathrm{~h}$. Whole lysate was immunoprecipitated with anti-human KIT Ab. After immunoblotting with 4G10, reprobing with anti-human KIT Ab was carried out.

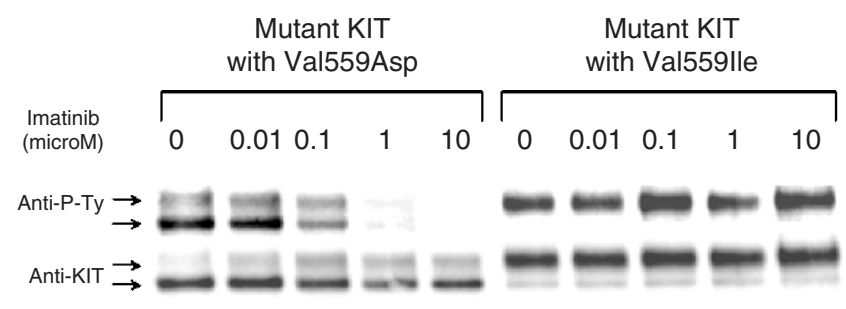

Figure 3 Effect of imatinib on autophosphorylation of mutant KIT. (a) Autophosphorylation of mutant KIT was examined after 90 min incubation at imatinib concentrations of $0,0.01,0.1,1$ and $10 \mu \mathrm{M}$ in HEK293 cells expressing Val559Asp or Val559lle. Autophosphorylation of mutant KIT with Val559Asp was almost completely inhibited at a concentration of $1 \mu \mathrm{M}$ of imatinib, but that with Val559lle was not even at an imatinib concentration of $10 \mu \mathrm{M}$. Whole lysate was immunoprecipitated with anti-human KIT Ab. Immunoblotting with 4G10 was carried out with subsequent reprobing with anti-human KIT Ab.

brane-type c-kit gene mutation of Val560Gly which is sensitive to imatinib, adult mast cell neoplasms usually have a TK II-type c-kit gene mutation which is resistant to imatinib. $^{7-9}$ We believe that this is the first ASM case reported with a juxtamembrane-type c-kit gene mutation. The juxtamembrane-type c-kit gene mutation is the most common type in GISTs, and simple amino-acid substitution (point

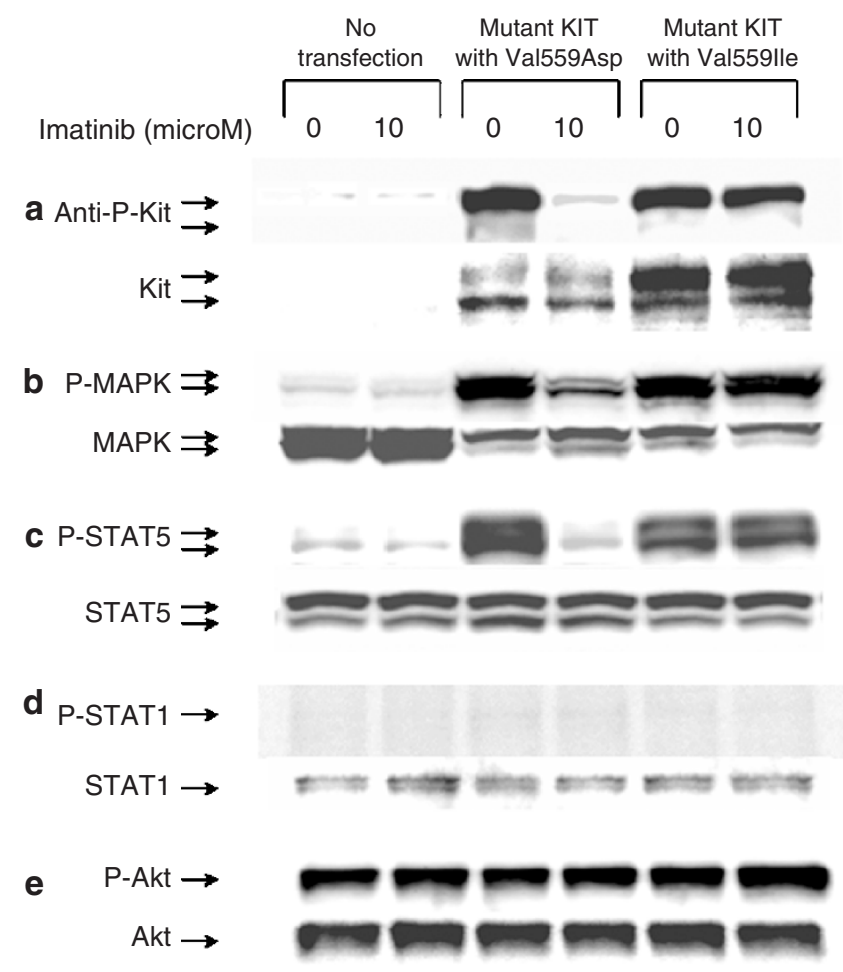

Figure 4 Effect of imatinib on phosphorylation of KIT downstream signaling molecules. (a-e) Autophosphorylation of KIT (a), phosphorylation of p44/p42 MAPK (b), STAT5 (c), STAT1 (d) and Akt (e) was examined at concentrations of 0 and $10 \mu \mathrm{M}$ of imatinib in HEK293 cells expressing Val559Asp or Val559lle. HEK293 cells without transfection were similarly treated as a control. When phosphorylation of mutant KIT with Val559Asp was inhibited by imatinib at a concentration of $10 \mu \mathrm{M}(\mathbf{a})$, that of MAPK (b) and STAT5 (c) was significantly inhibited. In contrast, not only KIT (a) but also MAPK (b) and STAT5 (c) remained phosphorylated in mutant KIT with Val559lle even after the addition of imatinib at a concentration of $10 \mu \mathrm{M}$. Apparent phosphorylation of STAT1 was not observed in all types of HEK293 cells even without imatinib treatment (d). Akt was strongly phosphorylated even in the original HEK293 cells, and the inhibitory effect of imatinib was not apparent in any type of HEK293 cells examined (e). The lysate was immunoblotted with anti-phospho-KIT, anti-phospho-p44/p42 MAPK, anti-phospho-STAT5, anti-phospho-STAT1 or anti-phospho-Akt Ab. Each membrane was reprobed with anti KIT, anti-p44/42 MAPK, anti-STAT5, anti-STAT1 or anti-Akt Ab.

mutation) of the codon 559 is often observed. Mutations of Val559Asp, Val559Ala or Val559Gly are usual types, and only one GIST case with Val559Ile has been reported to our knowledge. ${ }^{19-23}$ The juxtamembrane-type mutation observed in the present ASM case is unique. We characterized the mutation using HEK293 cells transfected with the mutated c-kit cDNA, and compared its properties to that of the GISTtype mutation, Val559Asp. The mutant KIT with Val559Ile was constitutively phosphorylated as well as that with Val559Asp, demonstrating that the c-kit gene mutation Val559Ile results in gain-of-function. However, the inhibitory effect of imatinib on autophosphorylation of the mutant KIT was different between the mutant KIT with Val559lle and that with Val559Asp. 
Imatinib almost completely inhibited the autophosphorylation of mutant KIT with Val559Asp at a concentration of $1 \mu \mathrm{M}$, but the autophosphorylation of mutant KIT with Val559Ile was not inhibited even at an imatinib concentration of $10 \mu \mathrm{M}$. The result indicates that the mutant KIT with Val559Ile was more resistant to imatinib than that with Val559Asp. It is well known that sensitivity to imatinib depends on the location of the c-kit gene mutation. Imatinib effectively inhibits KIT with juxtamembrane-type mutation, but fails to inhibit KIT with some TK II-type mutations, such as Val816Asp in mastocytosis. ${ }^{16,17}$ The phenomenon can be explained by the concept that some TK II-type activation loop mutations induce stabilization of the activation loop in an active conformation and/or structural alteration at the imatinib-binding site of KIT, resulting in a decreased affinity for imatinib. Since Ma et al ${ }^{18}$ reported that different TK II-type activation loop mutations at codon 816, such as Asp816Val, Asp816Tyr and Asp816Phe showed different sensitivities to imatinib, different amino-acid substitutions, even at the same codon, might cause the different imatinib sensitivities. In general, the juxtamembrane-type KIT mutations do not appear to show imatinib resistance. However, the structural change of the juxtamembrane domain clearly affects the structure of the kinase domains. Therefore, different amino-acid substitutions at the juxtamembrane non-activation loop region such as Val559Ile and Val559Asp might also cause different structural alteration and lead to different imatinib sensitivities. As the mechanism of the different inhibitory effects of imatinib on Val559Ile and Val559Asp mutations remains unclear, further examinations might be required to elucidate it, including substitutional experiment of codon 559 to various amino acids and structural analyses of those mutant KITs.

In the present study, we examined the effect of imatinib on downstream signaling molecules of KIT, using cells expressing mutant KIT with Val559Ile and mutant KIT with Val559Asp. Both transfectants showed apparent phosphorylation of MAPK and STAT5 but not STAT1, and phosphorylation of MAPK and STAT5 was inhibited by imatinib at a concentration of $10 \mu \mathrm{M}$ in cells expressing mutant KIT with Val559Asp but not in those with Val559Ile. As the inhibitory pattern by imatinib was similar to that of autophosphorylation of mutant KIT, KIT activation by the mutations appears to be closely related to the activation of MAPK and STAT5. The result suggested that both Val559Ile and Val559Asp mutations might activate Ras/MAPK and Jak/STAT signal transduction pathways.

In the present study, phosphorylation of STAT1 was not detected in cells expressing mutant KIT with Val559Ile nor those with Val559Asp. Ning et $a l^{25}$ reported that the STAT1 pathway plays an important role in KIT with TK II-type mutation. The STAT1 pathway might play an important role in TK II-type c-kit gene mutation but not in juxtamembranetype c-kit gene mutation.
A previous report showed that phosphorylation of Akt induced by SCF stimulation was inhibited by imatinib in a dose-dependent manner in M-07e cells expressing wild-type KIT. ${ }^{26}$ We also examined the phosphorylation status of Akt in the presence or absence of imatinib using original HEK293 cells and those expressing mutant KIT with Val559Ile or Val559Asp. However, Akt was apparently phosphorylated not only in cells expressing mutnat KIT with Val559Ile or Val559Asp but also in original HEK293 cells without KIT expression, and imatinib did not inhibit its phosphorylation in any types of cells used. As the PI3K/Akt signaling pathway was strongly activated in the original HEK293 cells even under the basal condition, it was not possible to assess the effect of c-kit gene mutations on phosphorylation of Akt by using HEK293 cells. The relationship between autophosphorylation of mutant KIT and phosphorylation of Akt should be investigated by transfection into other cells in which Akt is not strongly phosphorylated under the basal condition. Which down-stream signaling pathways are activated by various types of KIT mutations remains to be completely clarified.

Taken together, the Val559Ile mutation observed in ASM was proven to be of gain-of-function and imatinib-resistant. Recently, new tyrosine kinase inhibitors such as PKC412, dasatinib (BMS-354825), MLN518, AP23464 and AP23848 have been reported to exhibit inhibitory effect on imatinibresistant TK II-type activation loop mutations including Asp816Val with the STAT3 inactivation. ${ }^{27-30}$ There is a possibility that activation of KIT with Val559Ile mutation resistant to imatinib might be also effectively inhibited by those agents. However, it may not be inhibited because the mechanism of resistance might be different between the activation loop mutation and non-activation loop mutation. The effect of those drugs on the Val559Ile mutation remain to be examined.

\section{ACKNOWLEDGEMENT}

This study was partially supported by a grant from the Ministry of Education, Culture, Sports, Science and Technology of Japan (17013082).

1. Valent P, Hormy HP, Li C. Mastocytosis. In: Jaffe ES, Harris NL, Stein H, Vardiman JW (eds). World Health Organization Classification of Tumors: Pathology and Genetics of Tumors of Haematopoietic and Lymphoid tissues. IARC Press: Lyon, 2001, pp 293-301.

2. Hauswirth AW, Simonitsch-Klupp I, Uffmann M, et al. Response to therapy with interferon alpha- $2 \mathrm{~b}$ and prednisolon in aggressive systemic mastocytosis. Leuk Res 2004;28:223-224.

3. Valent $P$, Horny HP, Escribano L, et al. Diagnostic criteria and classification of masatocytosis: a consensus proposal. Leuk Res 2001;25:603-625.

4. Chabot B, Stephenson DA, Chapman VM, et al. The protp-oncogene c-kit encoding a transmembrane tyrosine kinase receptor maps to the mouse W locus. Nature 1988;335:88-89.

5. Zsebo KM, Williams DA, Geissler EN, et al. Stem cell factor is encoded at the $\mathrm{SI}$ locus of the mouse and is the ligand for the c-kit tyrosine kinase receptor. Cell 1990;63:213-224.

6. Maeda H, Yamagata A, Nishikawa $\mathrm{S}$, et al. Requirement of c-kit for development of intestinal pacemaker system. Development 1992;116:369-375. 
7. Furitsu $\mathrm{T}$, Tsujimura $\mathrm{T}$, Tono $\mathrm{T}$, et al. Identification of mutations in the coding sequence of the proto-oncogene c-kit in a human mast cell leukemia cell line causing ligand-independent activation of c-kit product. J Clin Invest 1993;92:1736-1744.

8. Nagata $\mathrm{H}$, Worobec $\mathrm{AS}$, Oh $\mathrm{CK}$, et al. Identification of a point mutation in the catalytic domain of the protooncogene c-kit in peripheral blood mononuclear cells of patients who have mastocytosis with an associated hematologic disorder. Proc Natl Acad Sci USA 1995;92:10560-10564.

9. Longley Jr BJ, Metcalfe DD, Tharp $\mathrm{M}$, et al. Activating and dominant inactivatingc-KIT catalytic domain mutations in distinct clinical forms of human mastocytosis. Proc Natl Acad Sci USA 1999;96: 1609-1614.

10. Hirota $S$, Isozaki K, Moriyama $Y$, et al. Gain-of-function mutations of c-kit in human gastrointestinal stromal tumors. Science 1998;279: 577-580.

11. Bittner C, Henz BM, Welker $P$, et al. Identification of activating c-kit mutations in adult-, but not in childhood-onset indolent mastocytosis: a possible explanation for divergent clinical behavior. J Invest Dermatol 1998;111:1227-1231.

12. Akin C, Fumo G, Yavuz AS, et al. A novel form of mastocytosis associated with a transmembrane c-kit mutation and response to imatinib. Blood 2004;103:3222-3235.

13. Lux ML, Rubin BP, Biase TL, et al. KIT extracellular and kinase domain mutations in gastrointestinal stromal tumors. Am J Pathol 2000;156:791-795.

14. Hirota S, Nishida T, Isozaki K, et al. Gain-of-function mutation at the extracellular domain of KIT in gastrointestinal stromal tumours. J Pathol 2001;193:505-510.

15. Kinoshita K, Isozaki K, Hirota S, et al. c-kit gene mutation at exon 17 or 13 is very rare in sporadic gastrointestinal stromal tumors. J Gastroenterol Hepatol 2003;18:147-151.

16. Ma Y, Zeng S, Metcalfe DD, et al. The c-KIT mutation causing human mastocytosis is resistant to STI571 and other KIT kinase inhibitors; kinases with enzymatic site mutations show different inhibitor sensitivity profiles than wild-type kinases and those with regulatorytype mutations. Blood 2002;99:1741-1744.

17. Zermati $Y$, De Sepulveda $P$, Feger $F$, et al. Effect of tyrosine kinase inhibitor STI571 on the kinase activity of wild-type and various mutated c-kit receptors found in mast cell neoplasms. Oncogene 2003:22:660-664.
18. Demetri GD, von Mehren $M$, Blanke $C D$, et al. Efficacy and safety of imatinib mesylate in advanced gastrointestinal stromal tumors. N Engl J Med 2002;347:472-480.

19. Taniguchi M, Nishida T, Hirota S, et al. Effect of c-kit mutation on prognosis of gastrointestinal stromal tumors. Cancer Res 1999:59:4297-4300.

20. Rubin BP, Singer S, Tsao $C$, et al. KIT activation is a ubiquitous feature of gastrointestinal stromal tumors. Cancer Res 2001;61:8118-8121.

21. Antonescu CR, Sommer G, Sarran L, et al. Association of KIT exon 9 mutations with nongastric primary site and aggressive behavior: KIT mutation analysis and clinical correlates of 120 gastrointestinal stromal tumors. Clin Cancer Res 2003;9:3329-3337.

22. Andersson J, Bumming P, Meis-Kindblom JM, et al. Gastrointestinal stromal tumors with KIT exon 11 deletions are associated with poor prognosis. Gastroenterology 2006;130:1573-1581.

23. Lasota J, Miettinen M. KIT and PDGFRA mutations in gastrointestinal stromal tumors (GISTs). Semin Diagn Pathol 2006;23:91-102.

24. Ohashi A, Kinoshita K, Isozaki K, et al. Different inhibitory effect of imatinib on phosphorylation of mitogen-activated protein kinase and Akt and on proliferation in cells expressing different types of mutant platelet-derived growth factor receptor-alpha. Int J Cancer 2004;111:317-321.

25. Ning ZQ, Li J, McGuinness $M$, et al. STAT3 activation is required for Asp(816) mutant c-Kit induced tumorigenicity. Oncogene 2001;20:4528-4536.

26. Heinrich MC, Griffith DJ, Druker BJ, et al. Inhibition of c-kit receptor tyrosine kinase activity by STI 571, a selective tyrosine kinase inhibitor. Blood 2000;96:925-932.

27. Growney JD, Clark JJ, Adelsperger J, et al. Activation mutation of human c-KIT resistant to imatinib mesylateare sensitive to the tyrosine kinase inhibitor PKC412. Blood 2005;106:721-724.

28. Schittenhelm MM, Shiraga S, Schroeder A, et al. Dasatinib (BMS354825), a dualSRC/ABL kinase inhibitor, inhibits the kinase activity of wild-type, juxtamembrane, and activation loop mutant KIT isoforms associated with human malignancies. Cancer Res 2006;66:473-481.

29. Corbin AS, Griswold IJ, La Rosee P, et al. Sensitivity of oncogenic KIT mutants to the kinase inhibitors LN518 and PD180970. Blood 2004; 104:3754-3757.

30. Corbin AS, Demehri S, Griswold IJ, et al. In vitro and in vivo activity of ATP-based kinase inhibitors AP23464 and AP23848 against activationloop mutants of Kit. Blood 2005;106:227-234. 